\title{
Study on A Novel ANFIS Controller for VSC-HVDC
}

\author{
Tian Yang ${ }^{1, a}$, Ran $\mathrm{Li}^{1, \mathrm{~b}}$ \\ ${ }^{1}$ Department of Electrical Engineering, North China Electric Power University, \\ Baoding, Hebei, China, 071003 \\ acathaywarrior@163.com, ${ }^{b} 978250509 @ q q . c o m$
}

Keywords: ANFIS; VSC-HVDC; PID

\begin{abstract}
Due to the PI control system for VSC-HVDC(Voltage Source Converter based High Voltage Direct Current) has problems of tuning parameters, excess number and so on, a novel controller composed of PID function and multiple-output Adaptive Neuro-Fuzzy Inference System (PID-ANFIS) is presented in this paper, which is made up of neural network, two order fuzzy control and PID control. The PID-ANFIS controller takes place of double loop PI control system, performing the functions of PI inner loop's fast tracking and decoupling as well as PI outer loop's variable transition. The simulation results show: the controller presented has significant improvement with faster speed, smaller overshoot and better robustness by comparison to PI and it is a viable choice for VSC-HVDC control system.
\end{abstract}

\section{Introduction}

Voltage Source Converter based High Voltage Direct Current(VSC-HVDC)is a nonlinear and strongly coupled system, which is usually controlled by PI in practical engineering.Several current researchs accept neural networks as VSC-HVDC controller ${ }^{[1-3]}$. This paper presents a novel controller composed of PID function and multiple-output Adaptive Neuro-Fuzzy Inference System (PID-ANFIS). PID-ANFIS is made up of neural network, two order fuzzy control and PID control, so that it is able to realize multiple-variable decoupling control.The MATLAB simulation results demonstrate that PID-ANFIS controller has a superior performance over conventional IP controller.

\section{VSC-HVDC model}

VSC-HVDC has the low frequency mathematical structure as below ${ }^{[4-5]}$ :

$$
\left\{\begin{array}{l}
L \frac{d i_{d}}{d t}+R i_{d}=u_{d}-v_{d}+w L i_{q} \\
L \frac{d i_{q}}{d t}+R i_{q}=u_{q}-v_{q}-w L i_{d} \\
C \frac{d V}{d t}=i_{d c}-i_{L} \\
P=1.5 u_{d} i_{d}, Q=-1.5 u_{d} i_{q}
\end{array} .\right.
$$

Where $R$ is the ac-side equivalent resistance of VSC converter , $L$ is the ac-side equivalent inductance, $C$ is the dc-side equivalent capacitance; $u_{d}, u_{q}$ present the voltage of power grid ac-side in dq reference coordinates, $v_{d}, v_{q}$ present the voltage of convertor ac-side in dq reference coordinates, $i_{d}, i_{q}$ present the current in dq reference coordinates; $V$ is the direct voltage of dc-side, $i_{d c}$ presents the dc-side current, $i_{L}$ presents the dc-side load current; $P, Q$ present the instantaneous power value. The last line of (1) means: when $u_{q}=0, P$ is only controlled by $i_{d}$ and $Q$ is only controlled by $i_{q}$ in steady state. The reason is dq transformation needs power grid voltage orientation. 


\section{ANFIS structure}

ANFIS controller is a nonlinear T-S fuzzy model(Sugeno fuzzy model), which is a new method to automatically generate fuzzy rules from numeric data sets. Inputs of ANFIS are based on artificial neuron with gaussian membership degree function and able to introduce blurring inwards.BP(back propagation) optimizing algorithm performs the function of adapting fuzzy rule parameters through connection weight learning so as to correct the shortcomings of fuzzy controller.The structure of ANFIS is the same as usual fuzzy neural network and has a kind of forward propagation.

T-S fuzzy model, the most novel fuzzy type, can be steadily analysed by Lyapunov Theory,so it correctify the problem of conventional fuzzy theory.ANFIS is made up by T-S fuzzy controller , neural network and linear consequent part.

If there are two fuzzy rules shown in (2),the structure of corresponding ANFIS can be shown as Fig.(1):

$R_{1}:$ if $x$ is A1, $y$ is B1 ;then $f_{1}=p_{1} x+q_{1} y+r_{1}$
$R_{2}:$ if $x$ is A2, $y$ is B2; then $f_{2}=p_{2} x+q_{2} y+r_{2}$

Where A1, B1, A2, B2 present the fuzzy subsets of inputs.

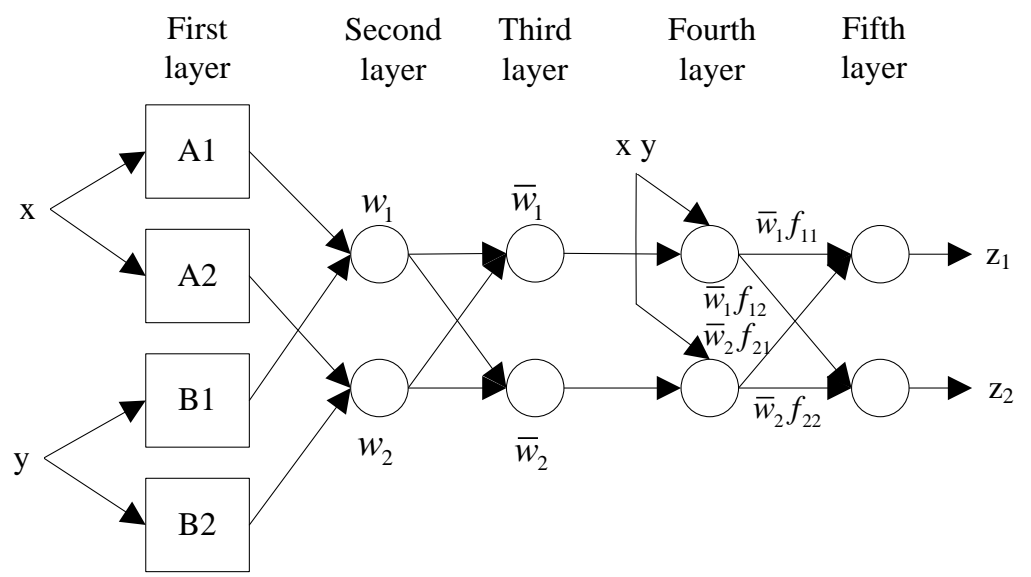

Fig. 1 ANFIS structure with two fuzzy rules

Fig.(1) shows that ANFIS has five layers. The function of each layer can be described in (3-7):

First layer (blurring): calculating the gaussian membership degree function value of every input $\mu_{i j}$, shown as (3). $i=1$, 2 presents the code of fuzzy rules; $j=1,2$ presents the code of first layer nodes; $\left\{b_{i j}, c_{i j}\right\}$ are parameters of antecedent parts, which have the information of the IF part.

$$
\mu_{i j}=e^{\left(-\frac{\left(x_{j}-c_{i j}\right)^{2}}{b_{i j}}\right)}
$$

Second layer: calculating incentive intensitis of antecedent parts, as (4). $w_{i}$ presents the incentive intensity of the $i_{\text {th }}$ fuzzy rule.

$$
w_{i}=\prod_{i=1}^{2} \mu_{i j}
$$

Third layer: normalizing incentive intensities by gravity method, as (5). $\bar{w}_{i}$ presents the normalized incentive intensity of the $i_{\text {th }}$ fuzzy rule.

$$
\bar{w}_{i}=\frac{w_{i}}{\sum w_{i}}
$$

Fourth layer: calculating outputs of every fuzzy rule, as (6). $f_{i}$ presents the consequent part output of the $i_{\text {th }}$ fuzzy rule. $\left\{p_{i}, q_{i}, o_{i}\right\}$ are parameters of consequent parts, which have the information of the THEN part.

$z_{i}=\bar{w}_{i} f_{i}=\bar{w}_{i}\left(p_{i} x+q_{i} y+o_{i}\right)$

Fifth layer: calculating the total output. $z_{r}$ presents the $r_{\text {th }}$ total output. 


$$
z_{r}=\sum z_{i}=\sum \bar{w}_{i} f_{i}
$$

\section{PID-ANFIS design}

ANFIS belonging to fuzzy neural networks, has the ability of infinite approximation to nonlinear equations in theory. ANFIS with multi-output possesses the same number of outputs with consequent parameters sets,so that it is able to realize multivariable static decoupling.

Usually, fuzzy controller has two inputs: one is the error between reference and practical output, the other is the differentiation of the frontal error. This forms the structure of two order fuzzy control,namely PD-fuzzy controller. Due to several existing analysis paper, PD-fuzzy controller has problems of too much fuzzy rules, fuzzy rules design and defective level of steady-state error.These problems need improvements.

In this paper,the process of establishing PID-ANFIS controller is:first, inventing PD-ANFIS by combining two order fuzzy control structure and ANFIS; then adding an integration module to PD inputs to form PID-ANFIS controller.This means PID-ANFIS has three inputs: one is the error between reference and practical output, another is the differentiation of the frontal error and the last one is the integration of the frontal error. Adding the integration of error will improve the level of steady-state error. PID parameters will be tuned by ANFIS inserted.As ANFIS has a reduced structure,more inputs can not lead to significant increase of information than fuzzy control.The structure of PID-ANFIS is shown as Fig.(2).

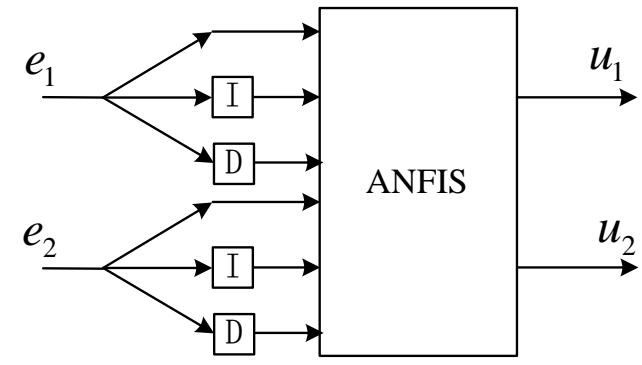

Fig.(2) PID-ANFIS structure

\section{Analysis of simulation examples}

This paper selects VSC-HVDC model from MATLAB Demos as the control object, inserts PID-ANFIS as the controller,while taking PI controller as a comparision. For considerations of tne wind power integration, the rectifier side of VSC-HVDC takes the PQ decoupling control method and the inverter side takes the VQ decoupling control method.The simulation results are shown as Fig.3.

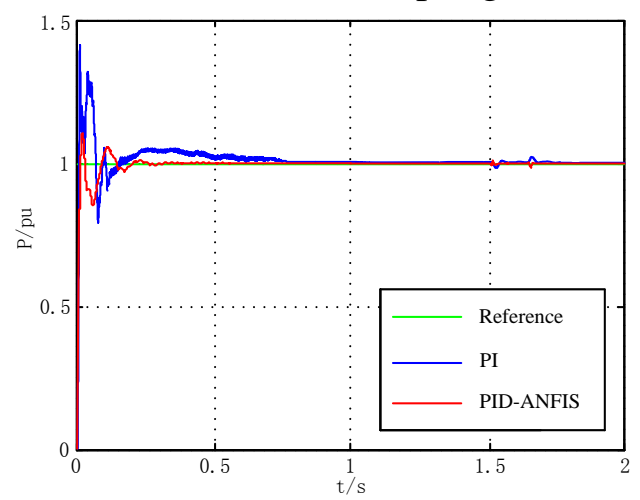

(a) Rectifier side P control

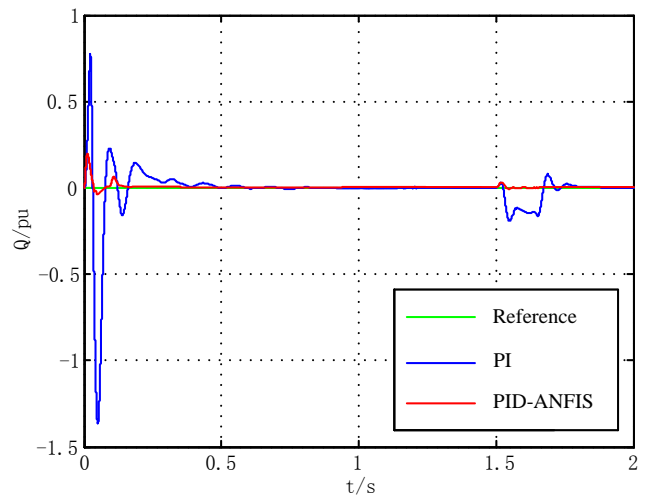

(b) Rectifier side Q control 


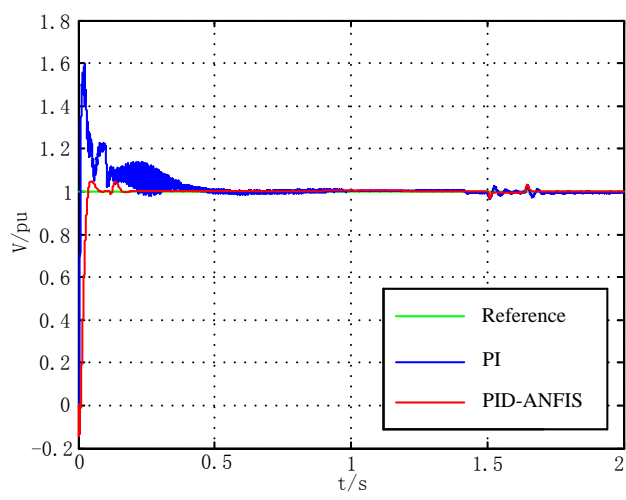

(c) Inverter side $\mathrm{V}$ control

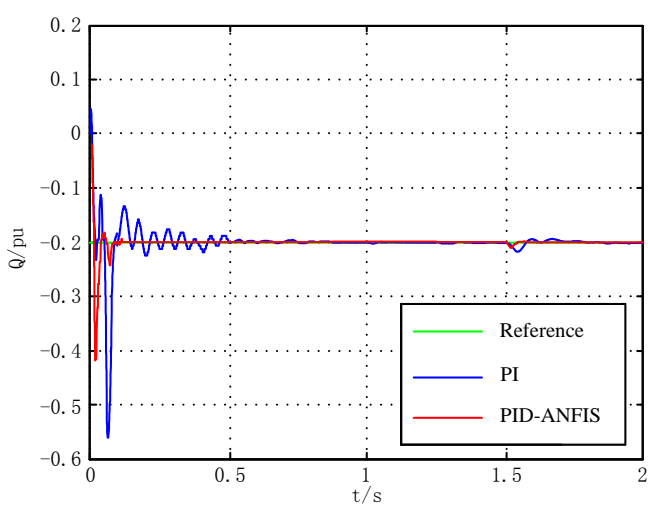

(d) Inverter side Q control

Fig. 3 Simulation results

The simulation is executed in two situations:

First, VSC-HVDC starting simulation, in which the active power reference is set as 1 , the reactive power reference is set as 0 , the dc-side voltage reference is set as 1 and the ac-side voltage is set as -0.2 .

Then, VSC-HVDC faulting simulation, in which the four references mentioned above are maintained. And the fault time is $1.5 \mathrm{~s}$, duration time is $0.2 \mathrm{~s}$.

In Fig.3, the green line presents the reference value, the red line presents the PID-ANFIS effect and the blue line presents the conventional PI effect. From Fig.3, PID-ANFIS has shown less overshoot and less response time than PI in the starting test. When $t=1.5 \mathrm{~s}$, there is a single phase short circuit happening at rectifier side and both controllers take effects to maintain stability. But PID-ANFIS spends less time and has less fluctuation amplitude than PI.

\section{Conclusions}

This paper studies the VSC-HVDC mathematical model and ANFIS structure, then presents a novel PID-ANFIS controller instead of PI. Simulation results show that VSC-HVDC controlled by PID-ANFIS can performs better in the starting and fault control test.

\section{References}

[1] LI Shuang, WANG Zhi-xin, WANG Guo-qiang. Application of an improved PSO-based PID neural network controller for VSC-HVDC [J]. Proceedings of the CSEE , 2013, 33(3):14-21.

[2] WANG Guo-qiang, WANG Zhi-xin. Application of PSO and PIDNN controller for VSC-HVDC [J]. Proceedings of the CSEE, 2011, 31(3):8-13.

[3] ZHANG Hai-yan. Fuzzy neural network controller for VSC-HVDC transmission system [J]. Electrical Measurement\&Instrument, 2010, 47(535):34-41.

[4] HOU Zhao-ran. Research on control method of PWM rectifier based on virtual flux orientation [J]. Power System Protection and Control, 2014, 42(21):105-109.

[5] SONG Guo-bing, RAN Meng-bing, JIN Xing-fu, et al. A novel protection method for VSC-HVDC transmission lines using single-end zero-mode current[J]. Power System Protection and Control, 2014, 42(15):1-7. 